\title{
Die Zeit des „unbekannten Patienten“ ist vorbei! Mit der Digitalisierung wächst die Informationsflut für Krankenhäuser
}

\begin{abstract}
Dr. Markus Müschenich, MPH, Facharzt für Kinderheilkunde, kennt das Krankenhausmanagement aus interner und externer Perspektive. Von 2009 bis 2012 war er im Vorstand der Sana Kliniken AG, zuvor leitete er mehrere Jahre die Geschicke des Vereins zur Errichtung evangelischer Krankenhäuser e.V. Seine berufliche Karriere startete er nach Abschluss seiner Assistenzarztzeit als Managementberater bei namhaften Unternehmen. Später gründete er selbst 2 Think Tanks. Sein Interesse gilt den digitalen Potenzialen des Gesundheitssystems. In seiner aktuellen Position als Co-Founder und Managing Partner der flyinghealth $\mathrm{GmbH}$ unterstützt er Gesundheits-Startups, die digitale Diagnose- oder Therapie-Anwendungen entwickeln, beim Eintritt in den 1. Gesundheitsmarkt. Er gilt als Vordenker für eine Medizin der Zukunft.
\end{abstract}

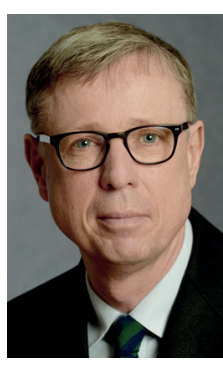

? Herr Dr. Müschenich, welchen Einfluss nehmen digitale Wissenspools auf die Diagnostik und Therapie im Krankenhaus?

Dr. Markus Müschenich: Das medizinische Wissen, das weltweit generiert wird, ist enorm. Es verdoppelt sich alle zwei bis fünf Jahre. Um die Informationen nachzuhalten und so aufzubereiten, dass Ärzte sie in ihre Entscheidung einbeziehen können, sind digitale Wissenspools unerlässlich. Ein Mensch schafft es nicht. Computer wie der Watson von IBM werten unstrukturiert zur Verfügung stehende Daten aus und leiten Entscheidungshilfen ab. Ob die
Computerempfehlungen den ärztlichen Einschätzungen standhalten, wird gegenwärtig an zwei Standorten in den USA getestet, für die Onkologie in Los Angeles und für die Neonatologie in San Francisco. Vermutlich werden in nächster Zeit Computer die Entscheidung des Arztes überprüfen. Bei der Digitalisierung geht es noch um einen weiteren Aspekt. Denn noch rasanter entwickelt sich die Menge an Patientendaten. Sie verdoppelt sich alle 73 Tage. Für einen Diabetiker fallen in vier Jahren rund 500000 Datensätze an. Dies sind nicht nur Parameter, die im Gesundheitssystem zwecks Diagnostik, Schulung, Einstellung und Kontrolle erhoben werden, sondern immer häufiger Daten, die der Patient selbst mitbringt.

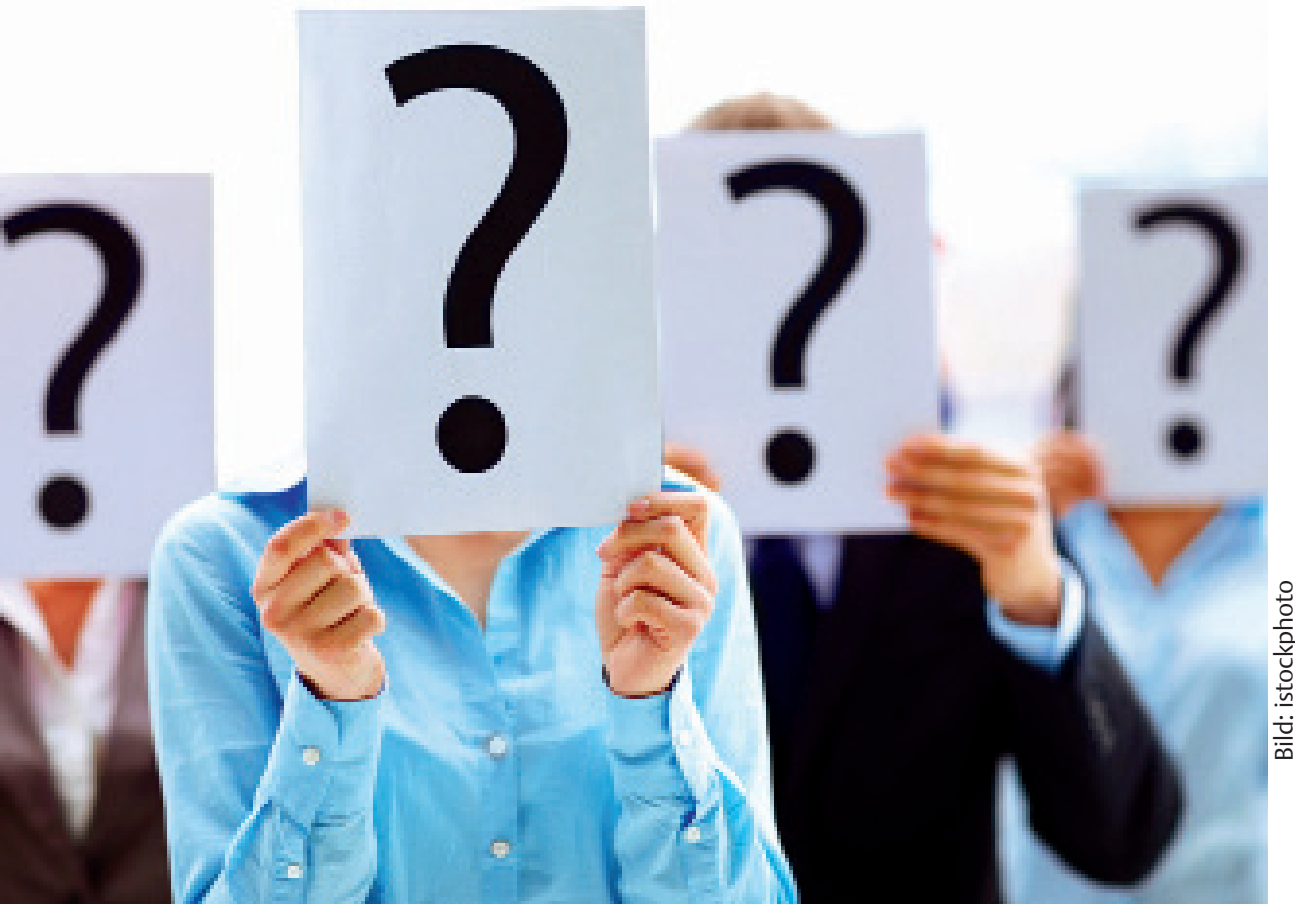

? Können Sie das an einem Beispiel veranschaulichen?

Müschenich: Patienten mit einer chronischen Krankheit beobachten ihren Krankheitsverlauf oft sehr genau und erheben eigene Messdaten, die sie in ihrer privaten elektronischen Patientenakte dokumentieren. Apple stellt hierfür seinen Kunden beim Kauf eines IPhones eine kostenlose App zur Verfügung. Die Nutzer erwarten natürlich von ihrem Arzt, dass er diese Daten bei den Therapieoptionen berücksichtigt. Gleiches gilt für schwangere Frauen, die den Arzt zurate ziehen, wenn sie zwischen einem Kaiserschnitt und einer natürlichen Geburt abwägen und nach einem geeigneten Entbindungsort suchen, dem unter Umständen eine Neonatologie-Station angegliedert sein muss. Für das Krankenhaus ist damit die Zeit des „unbekannten Patienten“ vorbei ist. Das heißt, Ärzte werden sich mit sämtlichen Informationen auseinandersetzen müssen, die der Patient über Monate und Jahre hinweg gesammelt hat. Im Schadensfall würde die Nichtberücksichtigung wohl haftungsrelevant werden.

\section{? Sind Krankenhäuser hierfür gerüstet?}

Müsschenich: Die Bedeutung elektronisch gestützter Prozesse haben alle Krankenhäuser erkannt, aber der Stand der Umsetzung ist unterschiedlich. Meines Wissens ist hierzulande noch kein Haus so weit, dass es die Informationen, die der Patient via App auf seinem Smartphone sammelt, direkt in die elektronische Patientenakte einspielen könnte. Das hätte jedoch enormes Potenzial für künftige Patientenkontakte. In der Mayo Clinic, die die elektronischen Eigendaten der Patienten bereits nutzt, schätzt man, dass sich infolge der Digitalisierung die Zahl der Patientenkontakte von derzeit 1,7 Millionen pro Jahr auf 200 Millionen 을 Kontakte bis 2020 nahezu verhundertfachen könnte.

? Wie könnte die Visite in 10 Jahren aussehen? 
Müschenich: Ich gehe davon aus, dass die digitale Medizin eine Aufgabenschiebung mit sich bringen wird. Wenn relevante Behandlungsinformationen in digitaler Form am Patientenbett zur Verfügung stehen und der virtuelle Kontakt zum Arzt bei Bedarf schnell hergestellt ist, lässt sich die Pflegevisite um medizinische Inhalte erweitern. Die Arztvisite wird stattdessen die gesamte Patientenkarriere samt der mitgebrachten Daten in den Blick nehmen. Vor allem werden sich Ärzte mit der Frage befassen müssen, welche ambulante Weiterbehandlung nach der Entlassung notwendig ist und wie sie die Kommunikation mit dem Patienten und dem nachbehandelnden Arzt gestalten.

? Was verändert sich in der Kommunikation mit Patienten?

Müschenich: Patienten werden selbstbewusster. Zwar sehen sie in dem Arzt nach wie vor den Experten, doch betrachten sie sich selbst als Kunde einer Dienstleistung und erwarten entsprechend mehr
Serviceorientierung. Sie möchten schnell, gut und zuvorkommend behandelt werden. Der Stand der Digitalisierung, das heißt, welche Möglichkeiten der Kommunikation und Akteneinsicht ein Krankenhaus bietet, auf welchen Wissenspool sich Ärzte stützen können und ob sie zusätzlich gelieferte Eigendaten in die Behandlung einbeziehen und dies auf elektronischem Wege unkompliziert möglich ist, wird damit zu einem relevanten Entscheidungsfaktor für die Krankenhauswahl, die ein Patient trifft.

? Wie gewichten Sie die Potenziale, aber auch die Risiken, die in der digitalen Medizin stecken?

Müschenich: Das größte Risiko wäre das Fehlen einer digitalen Medizin. Dies schadet sowohl dem Patienten, der bestmöglich behandelt werden möchte, als auch dem Krankenhaus als Wirtschaftsunternehmen, das auf Patientenzulauf angewiesen ist. Die digitale Schubkraft ist enorm. Sie wird neue Konzentrationsprozesse in der Krankenhauslandschaft hervorrufen, was punktuell mit Standortschließungen einhergeht. Das Risiko des Datenschutzes und der Datensicherheit halte ich verglichen damit für überschaubar, wenngleich es keinen 100\%igen Schutz vor krimineller Energie gibt. Worüber erst wenig gesprochen wird, ist jedoch die Gefahr der Entsolidarisierung in der gesetzlichen Versicherung, falls Gesundheitsinformationen beitragsrelevant werden sollten. Was passiert zum Beispiel, wenn ein Diabetiker seinen Lebensstil nicht ändert, obwohl ihm Folgekrankheiten prognostiziert werden. Drohen Schuldzuweisungen und Risikozuschläge? Welche Daten muss der Patient bereitstellen, welche darf er löschen? Hier gibt es noch viele Fragen, die einer öffentlichen Diskussion bedürfen.

Herr Dr. Müschenich, vielen Dank für das Gespräch!

Das Interview führte Dr. Adelheid Weßling, freie Journalistin, Düsseldorf. 